\title{
Mitigating the Reader Collision Problem in RFID Networks with Mobile Readers
}

\author{
Shailesh M. Birari and Sridhar Iyer \\ Indian Institute of Technology, Powai, Mumbai, India 400076. \\ (e-mail: \{shailesh,sri\}@it.iitb.ac.in)
}

\begin{abstract}
Radio Frequency Identification (RFID) is a means to identify and track objects using radio frequency transmission. An RFID system consists of readers and tags. Readers use radio signals to communicate with the tags. Tags may be active (battery powered) or passive (powered by the reader's signals). RFID is increasingly being used in many applications such as inventory management, object tracking, retail checkout etc. The reader collision problem occurs when the signal from one reader interferes with the signal from other readers. Such interference can result in lack of communication between the readers and some of the tags in the vicinity leading to incorrect and inefficient operation of an RFID system. This problem is further aggravated when mobile/hand-held readers are used in the system.

The evolving standards like ETSI EN 302208 for RFID readers use a CSMA based protocol called "Listen Before Talk" to reduce the reader collision problem. However, carrier sensing alone cannot solve the reader collision problem in RFID networks. For example, the signals from two readers that are out of range from each other, may still collide at the tags in the region between them. Although this aspect of the reader collision problem seems similar to the hidden terminal problem encountered in traditional wireless systems, existing solutions such as RTS-CTS mechanisms are not applicable. One of the reasons being: A reader may communicate with multiple tags simultaneously. Hence a seperate collision avoidance will be required between CTS sent by these multiple tags, making the protocol more complicated.

In this paper, we describe Pulse, a distributed protocol to reduce reader collisions. The operation of the Pulse protocol is based on periodic beaconing on a separate control channel by the reader, while it is reading the tags. The protocol functions effectively not only with fixed RFID readers but also with mobile RFID readers. We show, using simulation in QualNet, that using Pulse protocol, the throughput (overall read rate) is increased by as high as $60 \%$ as compared to "Listen Before Talk" (CSMA) and by $232 \%$ as compared to Colorwave. We also present an analytical model for our protocol in a single hop scenario.
\end{abstract}

\section{INTRODUCTION}

RFID, as a technique of automatic identification, is increasingly being used to identify and track objects through the supply chain in industries and manufacturing process[1]. An RFID system consists of an RFID reader, which is a transmitter/receiver module connected to an antenna, and a set of RFID tags, each of which is a low functionality microchip connected to an antenna[2]. A tag, which is generally attached to an object, typically stores information about the object. The reader uses radio waves to communicate with the tag and access this information. A tag may be active(powered by an external battery) or passive(powered by energy in the reader's signals). Passive tag uses the energy from the RF field of the reader for any on-chip computation and also for communication back to the reader. Since the signal from a passive tag to the reader is a reflected signal, the read range of a reader is very limited.

Making the readers mobile has some important advantages that are summarised as follows:

- Cost: Not all applications require "always-on"/real-time sensing of the item to be tracked. So a large deployment of fixed readers to cover the area is an overkill. For example, is it important to instantaneously sense the removal of a coke can in a retail store? Instead a periodic walk-through of mobile reader suffices in such situation. Also fewer mobile readers would suffice to cover the deployment area thus reducing the cost to a considerable extent.

- Convenience: Mobile readers require no wiring hassles or disruption of activities. Also mobile readers promote faster deployment of application and increases end user convenience.

Many applications require readers to operate in close proximity of each other. Due to proximity, the signals from one reader might interfere with the signals from other readers. This interference is called reader collision[3].

Reader to Reader interference arises when stronger signal from a reader interfere with the weak reflected signal from a tag. For example, in fig. $1, R_{1}$ lies in interference region of reader $R_{2}$. The reflected signals reaching reader $R_{1}$ from tag $T_{1}$, can easily get distorted by signals from $R_{2}$. Note that such interference is possible even when the read range of the two readers do not overlap.

Multiple reader to tag interference arises when more than one reader try to read the same tag simultaneously. In fig. 2, the read range of the two readers overlap. Hence the signals from $R_{1}$ and $R_{2}$ might interfere at tag $T_{1}$. In such case, $T_{1}$ can not decipher any query and the tag is read neither by $R_{1}$ nor by $R_{2}$. Due to reader collisions, $R_{1}$ will be able to read $T_{2}$ and $T_{3}$ but it may not be able to read the tag $T_{1}$. In such case, $R_{1}$ will indicate presence of 2 tags instead of 3 .

Apart from incorrect operations, reader collisions also result in reduction of the overall read rate of the RFID system. Hence reducing these reader collisions is essential. Morever this problem is aggravated in case of mobile/handheld readers.

Standard multiple access mechanisms cannot be directly applied to RFID systems due to the following reasons.

- FDMA: With FDMA, the interfering readers use different frequencies to communicate with the tags. Since the RFID tags do not have any frequency selectivity, they 


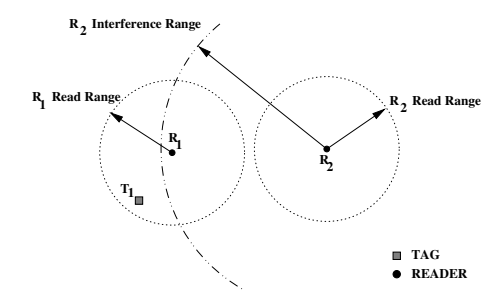

Fig. 1. Reader to Reader Interference

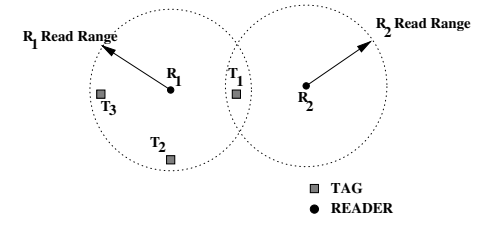

Fig. 2. Reader to Tag Interference

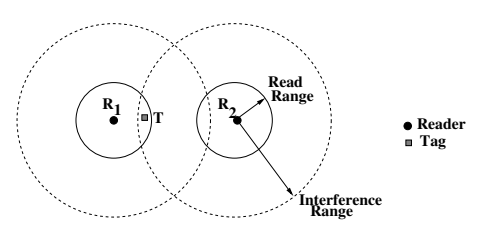

Fig. 3. Reader Collision making carrier sensing ineffective cannot select a particular reader frequency for communication. Hence FDMA is not a practical solution in RFID systems.

- TDMA: With TDMA, the interfering readers are alloted different time slots thus avoiding simultaneous transmissions. However this is similar to the well known coloring problem in graph theory[3] which is an NP-hard problem[3]. Also because of mobility, non interfering readers may move closer and start interfering. Hence a fixed TDMA based protocol may not be very efficient with mobility.

- CSMA: In fig. 3, the read ranges of the two readers donot overlap. However, the signals from reader $R_{2}$ can interfere with the signals from reader $R_{1}$ at tag $T$. This case can also happen when the two readers are not in each other's sensing range making carrier sensing ineffective in RFID networks.

- CDMA: CDMA will require extra circuitry at the tag which will increase the cost of the tags. Also code assignment to all the tags at the deployment site may be a complicated job. Hence CDMA may not be a cost effective solution.

FDMA, TDMA and CSMA are discussed in more detail in section II. Standard anti-collision protocols like RTS-CTS cannot be directly applied to RFID systems due to following reasons.

- In case of traditional wireless networks, only one node has to send a CTS back to the sender. However in RFID, if a reader broadcasts an RTS, all tags in the read range need to send back a CTS to the reader. This demands another anticollision mechanism for these CTS which will make the protocol more complicated.

- Also there are chances that a tag( say $T_{1}$ ) may not receive an RTS due to collision while other $\operatorname{tag}\left(\right.$ say $T_{2}$ ) may receive it. In such case, a CTS from $T_{2}$ is not a guarantee that there is no collision in the read range of the reader.

We propose a distributed protocol, Pulse, based on a beaconing mechanism. While a reader is reading the tags, it periodically broadcasts a beacon on a separate control channel. Any other reader that wants to communicate with the tags, first senses the control channel for a beacon. If it does not receive any beacon for a specified amount of time, it transmits a beacon and starts communicating with the tags. It continues to periodically transmit a beacon as long as it is communicating with the tags.

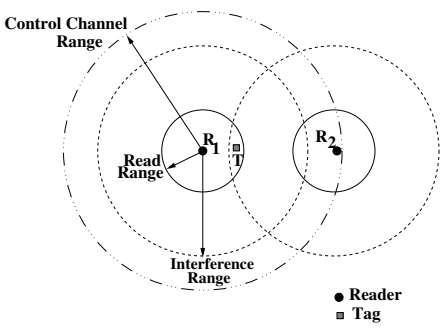

Fig. 4. Control Channel Range for Pulse Protocol

\section{RELATED WORK}

The Class 1 Generation 2 UHF standard[4] ratified by EPCGlobal[1] uses spectral planning(FDMA). It seperates the reader transmissions and the tag transmissions spectrally such that tags collide with tags but not with readers and readers collide with readers but not with tags. Such seperation solves the reader to reader interference since the reader transmissions and tag transmissions are on seperate frequency channels. However the tags donot have frequency selectivity. Hence when two readers using separate frequency communicate with the tag simultaneously, it will lead to collision at the tags. Thus multiple reader to tag interference still exists in this standard.

Colorwave[5] is a distributed TDMA based algorithm, where each reader chooses a random time slot to transmit. If it collides, it selects a new timeslot and sends a kick to all its neighbours to indicate selection of new timeslot. If any neighbour has the same color, it chooses a new color and sends a kick (small control packet) and this continues. If the percentage of successful transmission goes below certain threshold, the maxColors is incremented and if the percentage increases beyond certain threshold, the maxColors is decremented. However Colorwave requires time synchronisation between readers. Also, Colorwave assumes that the readers are able to detect collisions in the RFID system. However it may not be practical for a reader alone to detect the collisions that happen at the tags unless the tags take part in the collision detection.

ETSI EN 302 208[6] is an evolving standard being developed for RFID readers. It has a CSMA based protocol called "Listen Before Talk". The reader first listens on the data channel for any on-going communication for a specified minimum time. If the channel is idle for that time, it starts reading the tags. If the channel is not idle, it chooses a random backoff. However as described earlier, the readers may not be able to detect collision by carrier sensing alone.

\section{Pulse PROTOCOL}

RFID networks also suffer from the hidden terminal problem. As seen in figure $4, R_{1}$ and $R_{2}$ are not in each other's 
sensing region, but signals from $R_{2}$ might interfere with signals from $R_{1}$ at tag $T$. For such a scenario, a notification mechanism is required between $R_{1}$ and $R_{2}$ such that $R_{2}$ is informed of $R_{1}$ 's transmissions before it communicates with the tag. We propose to have this notification through a broadcast message called "beacon" on a seperate control channel.

The communication range in the control channel is such that, any two readers that can interfere with each other on the data channel (channel used to read the tags), are able to communicate on the control channel. Thus in fig 4 , since $R_{1}$ and $R_{2}$ interfere with each other on the data channel, they will be able to communicate on the control channel. This can be achieved by making the readers transmit at a higher power on the control channel than the data channel. The control channel can simply be a sub-band in the RFID spectrum apart from those used for reader-tag communication. Hence transmission on the control channel will not affect any ongoing communication on the data channel.

\section{A. Description}

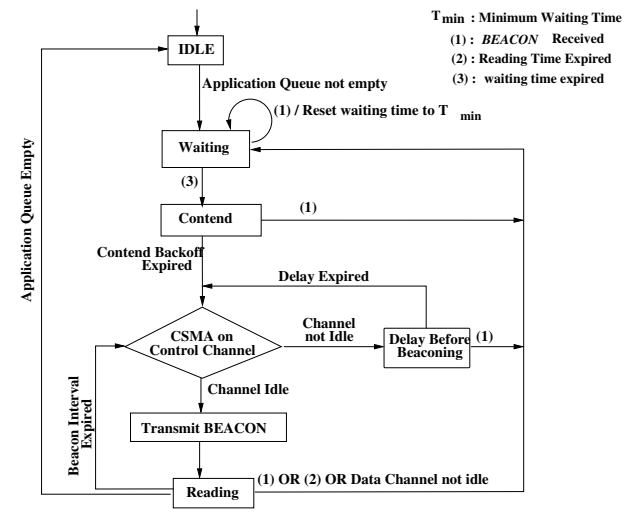

Fig. 5. Flow Chart for Pulse

Pulse protocol is present only at the reader since the tags do not take part in the collision avoidance. The data channel is used for reader-tag communication whereas the control channel is used for reader-reader communication. We assume that the reader is able to simultaneously receive on both the control and the data channel.

Following is an overview of the Pulse protocol.

- Before communicating with the tags, a reader has to wait in the state WAITING for a minimum time $T_{\text {min }}$ which is thrice the beacon interval. The time $T_{\min }$ is analogous to the DIFS time in 802.11 protocol[7]. Everytime it receives a beacon in this state, it resets its waiting time to $T_{\min }$.

- After $T_{\min }$ time has elapsed and it did not receiveany beacon for 3 that time, the reader concludes that there is no other reader in the neighbourhood which is reading the tags. Hence it enters a contention phase and chooses a random backoff time (contend_backoff) from the interval $[0 \ldots C W]$. If it chooses $i$, it waits for $i$ beacon intervals in state CONTEND. If it now receives a beacon, it has lost this cycle and waits for the next cycle, i.e until it does not receive a beacon for atleast $T_{\min }$ time. If the randomized backoff time is over and the reader did not receive any beacon, the reader assumes that there is no other reader compete and hence it sends a beacon on the control channel and starts communicating with the tags on the data channel. This randomized backoff helps to avoid collisions between readers, otherwise many readers would try to transmit the beacon simultaneously after waiting for $T_{\min }$ time. contend_backoff is a multiple of beacon intervals to improve fairness.

- While the reader is communicating with the tags, the reader sends a beacon on the control channel every beacon interval. This beacon acts as a notification to the neighbouring readers so that they can withhold their communication with the tags and thus avoid possible collisions. After the communication with the tags is over, the reader again waits in the WAITING state and the cycle continues.

- Everytime the reader sends a beacon, it first senses the control channel. If the control channel is busy, it continues to sense the control channel. As soon as the channel gets idle, the reader waits for a random delay(delay_before_beaconing) and senses the channel again to send the beacon. This random delay is a multiple of the beacon propagation delay and helps to avoid collisions otherwise many readers would simultaneously send the beacon after the channel became idle.

Fig. 5 shows the detailed flowchart and fig. 6 shows the detailed algorithm for the Pulse protocol.

The contend_backoff and the delay_before_beaconing in the protocol are similar to the backoffs in general wireless networks, they are decreased as long as the control channel is sensed idle, stopped when a transmission is detected, and reactivated when the control channel is sensed idle again. Also, if the reader receives a beacon during backoff (contend_backoff), in the contention phase, it stores the residual backoff timer and then waits for the next chance, i.e until it does not receive a beacon for atleast $T_{\min }$ time. It then uses this residual backoff time. This is done only to improve fairness amongst readers. Although the protocol seems to be simple, we show using simulations that it is effective in both static and mobile networks.

\section{Simulation EXPERIMENTS}

\section{A. Simulation Model}

We have simulated the UHF RFID network in QualNet with data channel frequency as $915 \mathrm{MHz}$ and the control channel frequency as $930 \mathrm{MHz}$. Our simulation model has the following assumptions: No interchannel interference between data and control channel, Free space propagation path loss, no fading, SNR based reception (SNR threshold $=10$, QualNet default), omni-directional antennas,negligible data processing and channel switching delay, $2 \mathrm{Mbps}$ data rate, $-91 \mathrm{dBm}$ Radio $\mathrm{Rx}$ sensitivity and $-81 \mathrm{dBm} \mathrm{Rx}$ threshold. The transmission power of the RFID node is adjusted to $-45 \mathrm{dBm}$, to make the read range $\sim 5$ feet as is the case with UHF RFID readers. We also assumed that packet collision is the only cause of packet loss. With these parameters the read range, sensing range and the interference range are 5.31 feet(1.62 meters), 17.71 
- CASE: Receive packet from application to send on the network

1: if state $=I D L E$ then

2: $\quad$ state $=$ WAITING

3: $\quad$ Set waiting_time_expired timer to $T_{\min }$

4: end if

- CASE: Control channel becomes busy

$1:$ if state $=$ CONTEND then

2: $\quad$ Pause contend_backoff_expired timer

3: end if

4: if state = DELAY_BEFORE_BEACONING then

5: Pause delay_before_beaconing_expired timer

6: end if

- CASE: Control channel becomes idle

$1:$ if state $=$ CONTEND then

2: $\quad$ Resume contend_backoff_expired timer

3: end if

4: if state $=$ DELAY_BEFORE_BEACONING then

5: $\quad$ Resume delay_before_beaconing_expired timer

6: end if

- CASE: BEACON Received

1: if state $=$ READING OR state $=$ CONTEND OR state $=$ WAITING then

2: $\quad$ Cancel all timers

3: $\quad$ state $=$ WAITING

4: $\quad$ Set waiting_time_expired timer to $T_{\min }$

5: end if

- CASE: Timer Expired

1: if waiting_time_expired timer AND state $=$ WAITING then

2: $\quad$ state $=$ CONTEND

3: $\quad$ Set contend_backoff_expired timer to previous residual value if any else select a new random backoff

4: end if

5: if (beacon_interval_expired timer AND state $=R E A D I N G)$ OR (contend_backoff_expired timer AND state $=$ CONTEND) then

6: if Control channel is IDLE then

7: $\quad$ transmit BEACON on control channel

8: $\quad$ Set reading_time_expired timer to max allowed communication time, if not set

9: $\quad$ Set beacon_interval_expired timer

10: $\quad$ state $=$ READING

$$
\text { Stse }
$$

state $=$ DELAY_BEFORE_BEACONING

end if

Set delay_before_beaconing_expired timer to random delay

6: end if

17: if reading_time_expired timer AND (state $=R E A D I N G$ OR state $=$ DELAY_BEFORE_BEACONING) then

18: cancel all timers

19: $\quad$ state $=$ WAITING

21: end if

Set waiting_time_expired timer to $T_{m i n}$

Fig. 6. Pulse Protocol Algorithm

feet(5.4 meters) and 23.29 feet(7.1 meters) respectively. Here the interference range is the maximum distance upto which a reader's transmission can interfere with another reader-tag communication. Thus the beacon range should be atleast equal to the interference range inorder to make this protocol effective.

We define the Beacon Range Factor(BRF) as the ratio of the control channel transmission power to the data channel transmission power. The power received at a receiver is inversely proportional to the square of the distance between the transmitter and receiver[8]. Thus BRF is given by [9]

$$
\mathrm{BRF}=\frac{P_{\text {Beacon }}}{P_{\text {Data }}}=\frac{r_{\text {Beacon }}^{2}}{r_{\text {Data }}^{2}}
$$

Thus with data range as 1.62 meters, inorder to have a beacon range of 7.1 meters, we require a BRF of 19.2.

\section{B. Performance metrics}

A query is said to be successfully sent if it is sent by a reader and is successfully received by all the tags in the read range i.e. it does not collide with any other query in the network. We define the system throughput and the percentage efficiency as follows.

\section{System Throughput $=$}

$\underline{\text { Total queries sent successfully(by all readers) }}$ Total time

\section{System Efficiency $(\%)=$}

$$
\frac{\text { Total queries sent successfully(by all readers })}{\text { Total queries sent(successful }+ \text { collided }) \text { by all readers }} \times 100
$$

In general, the tag identification is through a query-response protocol where the reader sends a query and the tag responds with its unique identification number. Higher the number of queries sent successfully, higher the throughput, and hence higher would be the number of tags identified by the readers. Percentage efficiency reflects the ability of a protocol to detect a possibility of collision at the tags and hence avoid unnecessary transmissions. An improvement in throughput indicate an improvement in the read rate whereas an improvement in the efficiency indicate reduction in collisions. Thus throughput and efficiency together define the effectiveness of the protocol. Through simulations we show that Pulse protocol is effective in both the dimensions.

\section{Simulation Scenarios}

We used the following simulation setup for running the experiments.

Tag setup: We used a field of 10 meter X 10 meter area, with 400 tags forming a grid of $20 \times 20$. The tags were placed throughout the simulation field with 0.5 meter interval so that most of the collisions in the field would be detected by these tags.

Fixed Readers:For fixed reader simulation, all the readers were randomly placed in the field. We used 20 random topologies with 3 different seeds in each case giving a total of 60 simulations per protocol.

Mobile Readers: For simulation of mobile readers, the initial placement of readers was a uniform grid of readers. We used a random way point mobility with low speed of 0.5 to 2 meters per second and 10 random seeds.

For simulation, the RFID application generated a packet(query) to be sent to the tags with exponential interarrival time of average $500 \mu$ sec throughout the simulation time of 60 seconds.

\section{Compared Protocols}

We compared our Pulse protocol with Aloha protocol, CSMA protocol[6] and Colorwave. A reader with Aloha protocol assumes that it is the only reader communicating with the tag. Hence when the reader wants to communicate with the tags, it simply starts its transmission without applying any collision avoidance. The CSMA protocol is similar to ETSI EN 302 208[6] with a listen time of $15 \mathrm{msec}$. For Colorwave protocol, we used the time slot of $10 \mathrm{msec}$. Rest of the experiment setup for Colorwave was as given in [5] We set the beacon interval of Pulse protocol as $5 \mathrm{msec}$ and $T_{\min }$ same as the listen time in CSMA i.e $15 \mathrm{msec}$. Using similar settings for both the protocols help us evaluate the MAC protocols in an unbiased manner. 


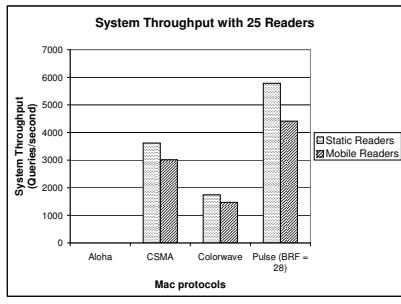

Fig. 7. Throughput comparison with 25 readers

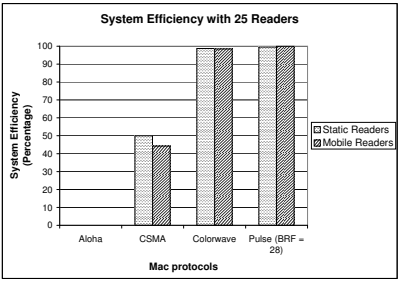

Fig. 8. Efficiency with 25 Readers

\section{RESULTS}

We first did a throughput comparison followed by efficiency comparison of Pulse with other protocols considering $\mathrm{BRF}=28$ and beacon interval $=5 \mathrm{msec}$. For each case we did the comparison initially on a 25 reader topology followed by topologies with different number of readers $(4 \ldots 64)$. We also studied the effect of BRF and beaconing interval on throughput and efficiency of Pulse in subsequent subsections.

\section{A. Throughput}

25 Reader Topology: Fig. 7 shows the comparison of Pulse with other protocols in 25 reader topology with static and mobile readers. As seen in the figure:

- With Aloha, almost every transmission in the system collided since the readers do not apply any collision avoidance.

- CSMA has better throughput than Aloha however the number of collisions using CSMA is still high because of the hidden terminal problem.

- Colorwave shows lower throughput because the timeslots are underutilised in a distributed timeslot mechanism.

- In Pulse, these collisions are avoided because the beacon sent by a reader acts as a notification to the neighbouring readers(including hidden nodes), which then withhold their transmission thus avoiding collisions. Pulse shows throughput improvement of $60 \%$ as compared to CSMA and $232 \%$ as compared to Colorwave in static topology.

- With mobility, the system throughput drops as compared to their static counterpart. However, Pulse still remains to be effective with throughput improvement of $46 \%$ as compared to CSMA and $200 \%$ as compared to Colorwave.

\section{B. Efficiency}

25 Reader Topology: Figure. 8 shows the percentage efficiency of the system using different MAC protocols.

- Efficiency with CSMA is nearly 50\% which means that $50 \%$ of the transmissions in the network get collided.

- Using Colorwave, the efficiency is almost $100 \%$ however, colorwave fails to give better throughput than Pulse.

- With Pulse, the efficiency is above $99 \%$ with both static and mobile readers. Thus Pulse is successful in detecting possibility of collisions and thus avoid the same.

Thus Pulse is definitely an improvment over the existing solutions in both the dimensions of throughput and efficiency. We further tested Pulse over different networks by varying the number of readers in the network and found that Pulse is effective even in highly dense mobile networks. We also studied the effect of the protocol parameters, BRF and beaconing interval, on the system throughput and efficiency. We found that $\mathrm{BRF}=28$ gives the highest throughput and change in beacon interval does not show any significant change in system throughput. Detailed results can be found in [9].

\section{Vi. Performance Modelling}

In this section we try to model our system inorder to find the average system throughput for a topology with static readers. We make the following assumption on the system to simplify the analysis.

- We assume a saturation case, i.e. all the readers always have to communicate with the tags.

- There are no hidden terminals on the control channel. Hence if a reader sends a beacon, all the readers receive the beacon. Note that even in such a case the readers might not be able to communicate with each other on the data channel since the range on the data channel is lesser than on the control channel.

- Since all the readers receive a beacon sent by a reader, normally there can be not more than one reader in the network communicating with the tags at any given point of time.

- We also assume that the time is slotted with the beacon interval $\left(T_{B I}\right)$ as the slot size, although in reality the time may not be slotted and synchronised across all nodes.

\begin{tabular}{|c|c|}
\hline variable & meaning \\
\hline$P_{s}$ & probability that $B D I$ contains successful transmission \\
\hline$B D I$ & Backoff Decrement Interval \\
\hline$E\left[T_{B D I}\right]$ & average duration of a BDI \\
\hline$E[B D I]$ & $\begin{array}{l}\text { average number of BDIs between two successful } \\
\text { transmissions by a reader }\end{array}$ \\
\hline$E\left[T_{\text {cycle }}\right]$ & $\begin{array}{l}\text { average duration between two successful } \\
\text { transmissions by a reader }\end{array}$ \\
\hline$T_{\text {read }}$ & $\begin{array}{l}\text { maximum duration for which a reader is allowed } \\
\text { to communicate with the tags at a time }\end{array}$ \\
\hline$\tau_{\text {query }}, \tau_{\text {beacon }}$ & $\begin{array}{l}\text { propagation delay on data and control channel } \\
\text { respectively }\end{array}$ \\
\hline$l_{\text {query }}, l_{\text {beacon }}$ & $\begin{array}{l}\text { transmission time of a query and beacon respectively } \\
\text { number of queries sent by a reader in } T_{\text {read }}\end{array}$ \\
\hline$S$ & $\begin{array}{l}\text { System throughput, number of queries tranmitted by } \\
\text { all the readers per unit time }\end{array}$ \\
\hline
\end{tabular}

TABLE I

NOTATIONS

Backoff Decrement Interval $(B D I)$ : The basis of our analysis is similar to as given in [10]. We define a backoff decrement interval $(B D I)$ to be the interval after which the backoff value is decremented. Fig. 9 shows the time line of 4 readers in the system whereas Fig. 10 shows the transmission of other readers $R_{2}, R_{3}, R_{4}$ superimposed on the timeline of reader $R_{1}$. Fig. 10 also shows the $B D I \mathrm{~s}$ as dotted lines. When reader $R_{1}$, at time $t_{2}$, receives a beacon from reader $R_{2}, R_{1}$ stops the backoff counter and resumes when it has not received any beacon for $T_{\min }$ time i.e at time $t_{3} . R_{1}$ then decrements its backoff value at the end of the next empty time slot at time $t_{4}$. Thus the $B D I$ duration is $T_{s}=T_{\text {read }}+T_{\text {min }}+1$. It 


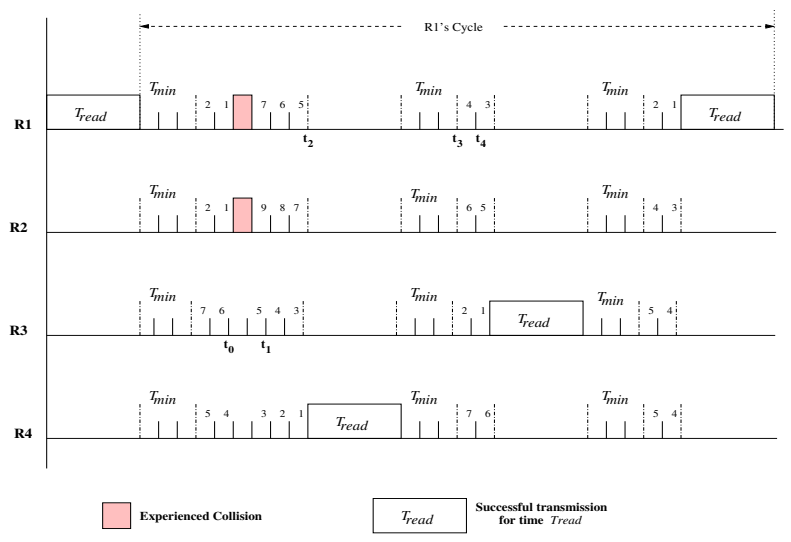

Fig. 9. Effect of transmissions on BDIs of other readers

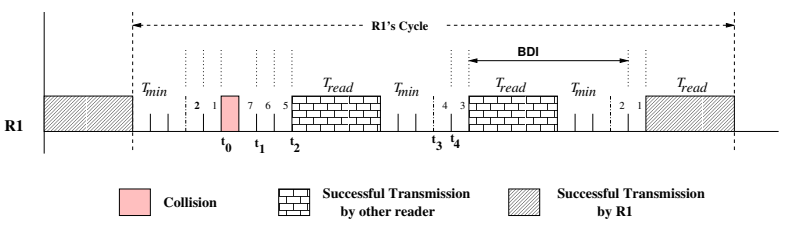

Fig. 10. BDIs of a Reader

may happen that more than one reader may decrement their backoff counter to 0 simultaneously in which case there will be a collision on the channel, (see $R_{3}$ 's time line at time $t_{0}$ in fig. 9). The duration of $B D I$ will then be from, $t_{0}$ to $t_{1}$ and thus $T_{c}=1$ (collision) +1 (empty time slot). If the $B D I$ contains neither a successful transmission neither a collision, then the duration of the $B D I$ will be a single empty time slot.

Cycle Duration: We define the read cycle of a reader to be the time between two successful channel captures by a reader. Note that successful channel captures in this case is different from successful transmissions. A reader captures the data channel for a duration of $T_{\text {read }}$. During a capture, the reader transmits multiple queries successively for $T_{\text {read }}$ time.

It may happen that more than one reader may decrement their backoff to 0 and hence might transmit and collide in which case each one will again choose a backoff value from $[0, C W]$ and transmit when the backoff counts down to 0 . This goes on until the beacon transmission is successful. Thus the expected number of $B D I$ s in a read cycle can be modelled by a geometric distribution and the expected duration of a cycle can be given by $E\left[T_{\text {cycle }}\right]=E[B D I] \times E\left[T_{B D I}\right]+T_{\text {read }}$

Throughput: Now, let us assume each reader is allowed to communicate with the tags for a maximum of $x$ beacon intervals, i.e. $T_{\text {read }}=x$ time slots. Now, each time slot in a $T_{\text {read }}$ will consist of one beacon transmission on the control channel and several transmissions(called queries) on the data channel by the reader. Thus each beacon interval $=\left(\tau_{\text {query }}+\right.$ $\left.l_{\text {query }}\right) \times($ no of queries in one beacon interval $)+\left(\tau_{\text {beacon }}+\right.$ $\left.l_{\text {beacon }}\right)$.

$$
\begin{gathered}
T_{\text {read }}=\left[\left(\tau_{\text {query }}+l_{\text {query }}\right) \times Q_{T_{\text {read }}}\right]+x\left(\tau_{\text {beacon }}+l_{\text {beacon }}\right) \\
Q_{T_{\text {read }}}=\left[\frac{T_{\text {read }}-x\left(\tau_{\text {beacon }}+l_{\text {beacon }}\right)}{\tau_{\text {query }}+l_{\text {query }}}\right]
\end{gathered}
$$

The average number of such successful periods $\left(T_{\text {read }}\right)$ by all the readers in one cycle is $P_{s} \times E[B D I]$ where $P_{s}$ is the probability that a $B D I$ contains a successful transmission. Thus the average number of queries sent by all the readers in one second, which is essentially the system throughput is

$$
S=\frac{Q_{T_{\text {read }}} \times P_{s} \times E[B D I]}{E\left[T_{\text {cycle }}\right]}
$$

Detailed derivation of this result and numerical validation of the performance analysis can be found in [9]

\section{CONCLUSION AND FUTURE WORK}

The reader collision problem in RFID networks is a hindrance for the proliferation of RFID. We presented a distributed protocol, Pulse, for an RFID network which uses a beaconing mechanism by sending periodic beacon on the control channel. Although the protocol is simple, we have shown that it mitigates the reader collision problem. It reduces the reader collisions to $1-2 \%$ and also increases the read rate of the system by as high as $98 \%$ as compared to CSMA. It requires very less overhead on the reader side and absolutely no support on the tag side. Our protocol is also very effective in a mobile scenario facilitating the use of mobile readers which is a cost effective solution for many applications.

We did not account for any channel switching delay in our simulations. However we believe it to be negligible as compared to the beacon interval. Ofcourse, the Pulse protocol demands for some extra circuitry on the receiver end of a reader. However Pulse protocol increases the throughput considerably. It also promotes the use of lesser number of readers by being effective in a mobile scenario. We believe this performance gain and reduction in number of readers required is high enough to offset the hardware modification required by this protocol.

Further research can involve porting of the Pulse protocol to readers with multiple data channels. Further analysis will lead to insights on the ideal parameters like the beaconing interval, waiting time and the maximum capacity of the protocol.

\section{REFERENCES}

[1] Electronic product code. http://www.epcglobalinc.org.

[2] Klaus Finkenzeller. RFID Handbook : fundamentals and applications in contactless smart cards and identification. Chichester : John Wiley, Leipzig, dritte edition, 2003.

[3] Daniel W. Engels. The reader collision problem. Technical report, epcglobal.org, 2002.

[4] Chris Diorio. Class 1 generation 2, uhf rfid. CTAN: www.autoid. org/SC31/2004/dec/SG3_200411_430_Gen2Update.pdf, December 2004.

[5] J. Waldrop, D. W. Engels, and S. E. Sarma. Colorwave: An anticollison algorithm for the reader collision problem. In IEEE Wireless Communications and Networking Conference (WCNC), 2003.

[6] ETSI EN 302 208-1,2 v1.1.1, September 2004. CTAN: http: //www . etsi.org.

[7] IEEE Computer Society LAN MAN Standards Committee. Wireless LAN Medium Access Control (MAC) and Physical Layer(PHY) Specifications. In ANSI/IEEE Std. 802.11, 1999 Edition, The Institute of Electrical and Electronics Engineers. New York, 1999.

[8] Prashant Krishnamurthy Kaveh Pahlavan. Principles of Wireless Networks. Pearson Education, 2002.

[9] Shailesh Birari. Mitigating the Reader Collision Problem in RFID Networks in Mobile Readers. Master's thesis, Indian Institure of Technology, Bombay, July 2005.

[10] O. Tickoo and B. Sikdar. Queing Analysis and Delay Mitigation in IEEE 802.11 Random Access MAC based Wireless Networks. In IEEE INFOCOM, 2004. 